\title{
Study Protocol: Screening and Treatment of Alcohol-Related Trauma (START) - a randomised controlled trial
}

\author{
Rama Jayaraj ${ }^{1,2^{*}}$, Mahiban Thomas ${ }^{3}$, David Kavanagh ${ }^{4}$, Peter d'Abbs ${ }^{1}$, Luke Mayo ${ }^{1}$, Valerie Thomson ${ }^{1}$, \\ Carolyn Griffin ${ }^{1}$ and Tricia Nagel ${ }^{1}$
}

\begin{abstract}
Background: The incidence of mandibular fractures in the Northern Territory of Australia is very high, especially among Indigenous people. Alcohol intoxication is implicated in the majority of facial injuries, and substance use is therefore an important target for secondary prevention. The current study tests the efficacy of a brief therapy, Motivational Care Planning, in improving wellbeing and substance misuse in youth and adults hospitalised with alcohol-related facial trauma.

Methods and design: The study is a randomised controlled trial with 6 months of follow-up, to examine the effectiveness of a brief and culturally adapted intervention in improving outcomes for trauma patients with at-risk drinking admitted to the Royal Darwin Hospital maxillofacial surgery unit. Potential participants are identified using AUDIT-C questionnaire. Eligible participants are randomised to either Motivational Care Planning (MCP) or Treatment as Usual (TAU). The outcome measures will include quantity and frequency of alcohol and other substance use by Timeline Followback. The recruitment target is 154 participants, which with $20 \%$ dropout, is hoped to provide 124 people receiving treatment and follow-up.
\end{abstract}

Discussion: This project introduces screening and brief interventions for high-risk drinkers admitted to the hospital with facial trauma. It introduces a practical approach to integrating brief interventions in the hospital setting, and has potential to demonstrate significant benefits for at-risk drinkers with facial trauma.

Trial Registration: The trial has been registered in Australian New Zealand Clinical Trials Registry (ANZCTR) and Trial Registration: ACTRN12611000135910.

Keywords: Facial trauma, Indigenous Australians or Aboriginal and Torres Strait Islanders, Alcohol related injury, Culturally appropriate intervention

\section{Background}

Globally, alcohol causes $3.2 \%$ of all deaths, or 1.8 million deaths annually, and accounts for $4.0 \%$ of disease burden [1]. Alcohol-related injuries are a problem in both high and low-income countries [2], including. Alcohol-related trauma is recognised as a major public health problem in Australia [3]. Alcohol abuse is a major contributor to the

\footnotetext{
* Correspondence: Rama.Jayaraj@menzies.edu.au

${ }^{1}$ Wellbeing and Preventable Chronic Diseases Division, Menzies School of Health Research and School of Environmental and Life Sciences, Charles Darwin University, Darwin, Northern Territory, Australia

${ }^{2}$ School of Environmental and Life Sciences, Charles Darwin University, Darwin, Northern Territory, Australia

Full list of author information is available at the end of the article
}

incidence of traumatic injury [4-7]: $27 \%$ to $47 \%$ of trauma patients test positive for alcohol use at the time of admission, and $30-40 \%$ test positive for other substance use [8]. Alcohol and other drug abuse induce physical and cognitive impairment that increases vulnerability to both unintentional injury and violence $[9,10]$. The impact of alcohol also extends to criminal offenses. An estimated $50 \%$ of all Australian offenders detained by police in 2007 for disorder and violent offences had consumed alcohol in the 48 hours prior to their arrest [11]. In 2004-2005, the cost of alcohol-related injury in Australia was estimated at AUD15.3 billion, when costs associated with crime and 
violence, treatment, loss of productivity and premature death were all included [12].

Australian Aboriginal and Torres Strait Islanders have high rates of injury, of hospitalisation and death in these people are caused by assault [13]. Alcohol represents a significant contributor to this increased risk [14].

The current research is conducted in Northern Territory (NT), which is situated in central and northern central Australia. It has a small population that is primarily located in two cities (Darwin and Alice Springs) and has extremely remote and sparsely populated areas. Between 2004 and 2009 , the NT had the highest rate of per capita alcohol consumption in Australia (15 litres of ethanol) [15,16], and the highest estimated rate of alcohol-related hospitalisations for assault. The incidence of alcohol-attributable deaths in NT from 1990 to 2002 was 0.64 per 100,000 population, compared with 0.21 per 100,000 population nationwide [14]. The annual total cost to the NT from alcohol, tobacco and illicit drug abuse in 2009 was estimated at AUD642 million, or $\$ 4,197$ per person [12].

The NT Aboriginal population is particularly at risk of alcohol-related harm and death, with 1.86 alcoholattributable deaths per 100,000 people, compared with 0.38 in other NT residents [14]. Violence is the most common cause of hospital admission for injury in the NT, accounting for $38 \%$ of injury admissions for Aboriginal people [17]. Aboriginal prisoners are also vastly overrepresented in the NT, representing $82 \%$ (850) of the daily average prison population, but only $32 \%$ of the NT population [18]. Evidence of links between assault and alcohol misuse is scant, but reports from offenders clearly link alcohol in violent assaults and other crime [19].

A particular focus of alcohol-related violence involves mandibular fractures [20]. Facial fractures in the NT are close to 120 per 100,000 of population, and in Indigenous people occur at a massive 155 per 100,000 [21]. Personal assaults, fights, and violence account for $91 \%$ of all facial traumas in the NT [21]: $72 \%$ of these patients are injured by an intoxicated person when they were also intoxicated, and another $8 \%$ are by an intoxicated person when they were sober. Most assaults against women in remote NT communities are alcohol-related, and are perpetrated by a husband or other family member [22].

In the general population, screening and brief counselling can be effective in reducing alcohol intake and assault associated with binge drinking [23-26]. While there is abundant evidence that brief interventions are effective in the treatment of high-risk drinking [27], there is less research on the impact of brief interventions on alcohol-related violence [20].

The current project breaks new ground, in examining the impact of a brief inpatient intervention for alcoholrelated facial trauma in a predominantly indigenous sample. In taking on this challenge, the cultural context of the intervention must be considered. A brief treatment that was specifically developed for use with indigenous Australians is Motivational Care Planning (MCP; Nagel et al. 2009). MCP incorporates key principles of several brief therapies: motivational interviewing, goal setting and problem solving. Motivational interviewing has been developed and used successfully as treatment for substance misuse and co morbidity with individuals and families [28-30]. In MCP, clients are encouraged to consider their life as a whole, rather than only focusing on the substance use, reviewing things that keep them strong, and take away their strength. A tree is used as a metaphor, and potentially affected domains (e.g. being on their land, spirituality, family) are presented pictorially. Among aspects that may take away strength is substance use, and clients are encouraged to consider the role this has in the overall picture.

Those who wish to make a behavioural change are encouraged to adopt a potentially achievable goal, and identify concrete steps toward it (represented as footsteps on a football field). Goal setting is well established as a strategy to guide effective self-management in a range of settings [31,32], and indigenous clients readily identify with the concept and metaphor adopted here. Potential issues are in achieving the initial steps are identified, and problem solving strategies are applied to these challenges [33]. In the current project, MCP is adapted to incorporate the nature of relationships between substance use and mandibular injury, while retaining a whole-of-life perspective.

\section{Aims of the study}

The study aims to conduct a randomised controlled trial to compare the impact of a culturally adapted brief intervention (MCP) and of standard care, with patients hospitalised with alcohol-related trauma. The primary assessed outcomes include alcohol and other substance use and distress, which are examined at Baseline and 6 months. Incidence of further injuries is tracked, but the current study is not powered to detect differential changes in this index.

\section{Design}

This study is a parallel, randomised controlled trial to evaluate the effectiveness of a brief culturally adapted intervention in improving outcomes for high-risk drinkers admitted to hospital with facial trauma. The expected flow diagram for the study is shown in Figure 1.

\section{Recruitment}

The study sample is opportunistically selected from patients who are admitted to the maxillofacial unit at Royal Darwin Hospital, in the Northern Territory of Australia. All at-risk drinkers admitted with facial 


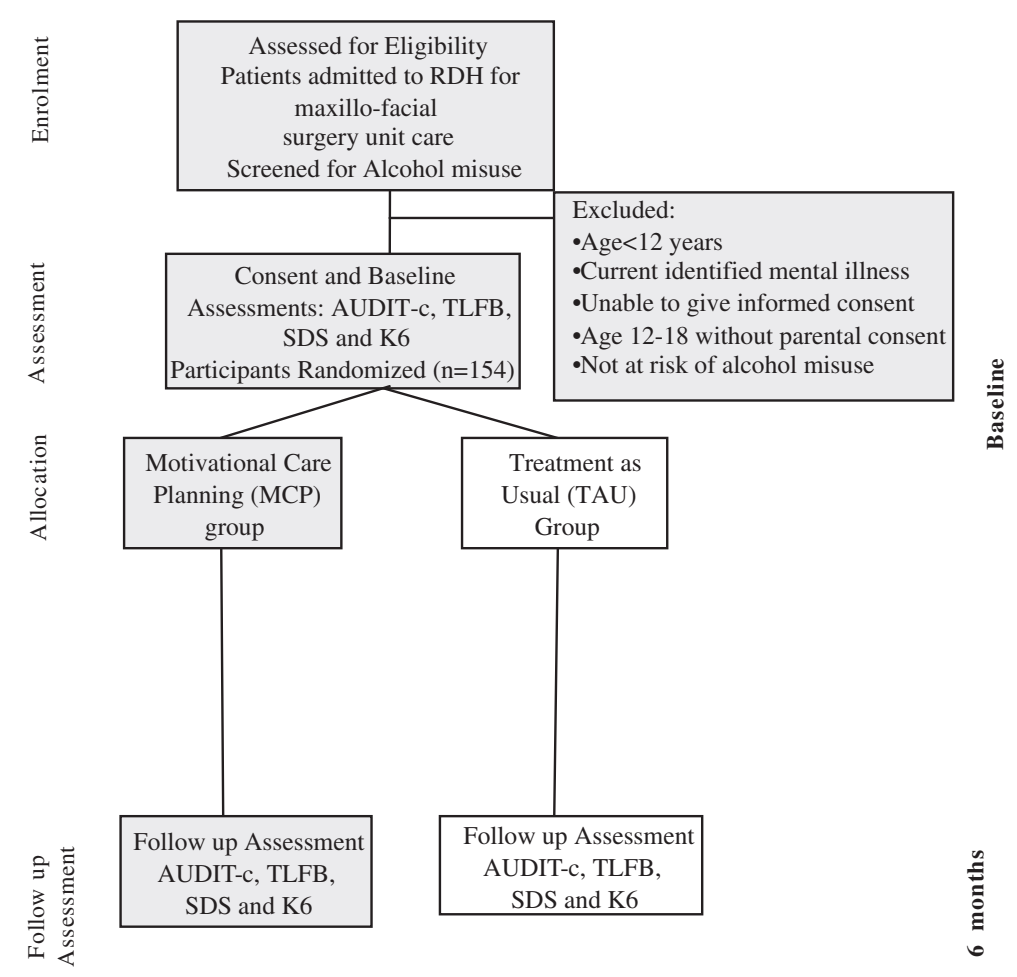

Figure 1 Expected CONSORT diagram.

trauma are assessed to determine their eligibility for inclusion. The hospital staffs assist the research team to identify potential participants.

\section{Inclusion/exclusion criteria}

Inclusion and exclusion criteria ensure that the sample can engage with interventions and assessments [34].

All participants must satisfy the following criteria at study entry:

1. An inpatient of Royal Darwin Hospital with facial trauma.

2. At least 12 years of age.

3. Identified at risk of alcohol misuse as measured by the Alcohol Use Disorders Identification Test (AUDIT-C $\geq 6$ ) $[35,36]$.

4. Able to give informed consent (and if less than 18 years of age, parental consent is provided).

\section{Assessment}

Assessments combine standardised measures and semistructured interviews with individuals and family members who provide care or support. At Baseline, a demographic questionnaire gathers age, gender, location of residence in the NT, stressors protective factors including reviewing strengths, stressors, family and support networks and amount and frequency of substance misuse. Data is collected by trained Indigenous and non-
Indigenous research officers and recorded on standardized forms. The screening and assessment tools have been presented in pictorial adaptation to the Aboriginal and Torres Strait Islander cultural and language context. The chosen measures (AUDIT-C, Kessler 6 [37]) have been tested in our previous work with Aboriginal and Torres Strait Islander people. They were found to be acceptable and are well understood.

\section{Screening instruments \\ AUDIT-C}

The Alcohol Use Disorders Identification Test (AUDIT) is the quick estimate of alcohol consumption and designed to detect hazardous and harmful levels of alcohol consumption [38]. This gold-standard screening test is developed by the World Health Organization (WHO) as a simple method of screening for excessive drinking and to assist in brief assessment. The AUDIT is widely used as a universal screening tool for emergency department and primary health care patients in USA and UK $[26,39]$. The AUDIT has been used to assess risky alcohol use in an Indigenous population in Queensland, and has shown to perform well [40]. The AUDIT-C, a brief version of the AUDIT, consists of three items. This version has been shown to have similar sensitivity and specificity to the full questionnaire [41]. The third question of the AUDIT-C alone (which examines the frequency of respondents had 6 or more drinks) predicts alcohol- 
related morbidity [42]. Weekly binge drinking has high specificity (from $79 \%$ to $96 \%$ ) and sensitivity (5\% to $83 \%$ ) [42-46]. Inclusion of this question in the current study is important, since consumption more than four drinks on a single occasion more than doubles the relative risk of an injury in the next 6 hours [47]. In the current study, data from the AUDIT-C are also used to assess alcohol consumption in cases where a full Timeline Followback measure (cf. below) cannot be obtained. The extent this abbreviated assessment is employed will be reported.

\section{Severity of Dependence Scale (SDS)}

$S D S$ is a 5 -item scale which focuses on the psychological aspects of dependence such as impaired control over drug use. It is a brief, easily administered instrument that is a reliable and valid screening tool in different cultural settings [48], in the context of dual diagnosis $[49,50]$, and across different substances [51]. It is used to screen for presence of substance-related disorder in this study, using a cut off of 3 [51].

\section{Outcome measures \\ Timeline Follow back (TLFB)}

The TLFB is a retrospective assessment of substance use, which employs recall of activities and events to cue estimates of consumption. Its use for assessment of alcohol consumption has been evaluated with clinical and non-clinical populations [52] and can generate precise information about patterns and variability [52]. In the current study, the TLFB is used to assess both alcohol and cannabis use over a 14-day period. After data is obtained on the 14-day period, participants are asked about whether that period was typical of their recent substance use. Estimates of their more typical use are recorded, and are subjected to secondary analyses.

\section{Kessler distress scale}

Kessler-6 (K6) is a 6-item version of the Kessler-10 (K10) measure of emotional distress [53]. The K10 is one of the consumer measures mandated for use in Australian mental health services, and has been validated among Australians with substance misuse [54]. The K6 is also highly predictive of mental disorder [55], showing a sensitivity of .85 and a specificity of .78 [56], compared with a sensitivity of 0.78 and specificity of 0.74 for the K10 [54]. Both the K10 and K6 have been used with Indigenous people in population surveys. In the current trial, $\mathrm{K} 6$ is used to assess psychological distress during the preceding month.

\section{Secondary outcome measures}

File Audits: Hospitalisations for alcohol-related injuries and illness in the preceding two 6-month periods are determined from the patients' files at Baseline and at 12 months post-treatment. In both cases, any mention alcohol being associated with an injury or illness is coded positive. We also code for any record of screening or assessment of alcohol use, distress or trauma over the period, and for any related intervention.

\section{Procedure}

Eligible trauma patients are identified in the maxillofacial unit and referred to the research staff, who obtain informed consent. All eligible participants are screened for high risk drinking. Full assessment of those screened at risk is performed prior to random allocation. Those at risk are randomly allocated to Motivational Care Planning or Treatment as Usual conditions. A statistician who is not directly involved in the analysis of the study results prepares the randomisation code to ensure that an approximate balance between in numbers is maintained between groups throughout the study. Allocations are concealed until the person's baseline assessment is completed. Sealed envelopes contain the sequences, and the use of the envelopes is monitored. Blinding and equipoise are strictly maintained through clear protocols, assessor training, and oversight of procedure by the Principal Investigator.

\section{Motivational Care Planning (MCP)}

Elements of Motivational Care Planning are described above and in previous publications [57]. MCP was developed in collaboration with Aboriginal Mental Health Workers, and differs from established approaches by inclusion of pictorial tools and a holistic, family focus. The 30-minute intervention is manualised and is usually completed in a single session, and is delivered by both Indigenous and non-Indigenous mental health research staff. A non-Indigenous version of MCP is used for the nonIndigenous participants. It omits aspects of primary interest to Indigenous people (e.g. hunting and gathering).

\section{Treatment as Usual (TAU)}

Participants randomised to TAU receive facial trauma treatment according to usual practice at the hospital, with addition of an information sheet on alcohol and trauma that was prepared in consultation with the project's Expert Reference Group.

\section{Fidelity of the intervention}

Therapists undertake a 2-day workshop on delivery of the manualised interventions, together with 3-monthly booster training and fortnightly supervision sessions to maintain fidelity. A sample of sessions are observed and rated by research investigators using a checklist of key features. Regular feedback on fidelity is given, with suggestions on how to adjust delivery. 


\section{Follow up assessment}

Face-to-face follow-up assessments are conducted at 6 months post-baseline, by researchers who are blind to condition. These assessments are conducted in the Royal Darwin Hospital, or in the closest health centre to the participant. In the case of people who cannot be contacted for face-to-face assessment, assessments are undertaken by telephone. Where participants cannot initially be contacted for follow-up assessments, attempts to contact them continue for up to 12 months post-baseline.

\section{Predictions}

Primary predictions

We predict that:

1. High-risk drinkers with maxillo-facial injuries who receive Motivational Care Planning will have greater reductions in (a) alcohol, on the TLFB and AUDITC; (b) other substance use on TLFB and (c) distress on K6, than participants receiving Treatment as Usual.

2. Greater improvements from Motivational Care Planning will be maintained to 6 months posttreatment.

\section{Secondary predictions}

Patients receiving Motivational Care Planning are expected to have reduced readmission rates for injury, although the study may be insufficiently powered to detect this effect.

\section{Sample size}

Assuming equality between conditions at Baseline, the study is powered to detect a differential reduction in alcohol consumption of $0.50 \mathrm{SD}$ in weekly alcohol consumption [58]. We argue that this is the minimum difference of any clinical significance. Setting the power at 0.80 and alpha at $0.05,62$ participants are required in each condition. While data will be analysed by intention to treat, the sample size allows for $20 \%$ attrition, deriving a target baseline sample of 154 .

\section{Analysis}

Continuous outcome variables are assessed for normality prior to analysis and transformed if necessary. Primary analyses use Linear Mixed Models analyses comparing the 2 conditions (MCP vs TAU) over 2 occasions (Baseline, 3 and 6 months post-baseline), allowing the analysis of data on all participants allocated to conditions at Baseline (i.e. intention to treat).

\section{Feasibility}

A total of more than 250 patients are admitted to the Royal Darwin Hospital maxillofacial unit each year. We anticipate that $62 \%$ of these inpatients will fulfil eligibility criteria for inclusion and that 77 patients per year will be eligible for randomisation over the 12-month recruitment period.

In order to minimise drop out we will undertake the following actions:

- Obtaining at least 3 means of contacting participants at recruitment (Telephone number of participant, telephone number their carers -family member and participants address);

- Following up by phone to update contacts and residential details every month;

- Liaising with the surgical team to link follow-up assessments with client outpatient visits.

\section{Consent and culturally appropriate approach}

Research officers will use pictorial and plain English information sheets, screening and intervention tools to assist understanding. Aboriginal and Torres Strait Islander participants will be offered communication support (interpreters, translators, assessed communication and inclusion of a support and cross-cultural family member or person of their choice). All information will be presented to participants by Indigenous researchers in a culturally appropriate manner, and written assessments will be administered orally in cases of limited literacy.

\section{Ethics and confidentiality}

The study has been granted ethical approval by the Human Research Ethics Committee of Department of Health and Families and Menzies School of Health Research. The approval number is HREC 2010-1438. Electronic data is password-protected, and identifying data is kept on a separate database from outcome data, allowing de-identification at the end of data collection. Assessment data will be accessible only to the investigators and support team.

\section{Discussion}

This project will introduce a practical approach to integrating brief interventions into the hospital setting, and has the potential to demonstrate significant benefits for at-risk drinkers with facial trauma. Findings from this project are expected to inform hospital-based treatment and secondary prevention of alcohol-related injury, not just in indigenous people in NT, but in other trauma treatment units throughout the world.

\section{Competing interests}

The authors declare that they have no competing interests.

\section{Authors' contribution}

JR conceived of the study, participated in its design and coordination, carried out the review, and drafted the manuscript. MT, DK, VT, CG, LM and $\mathrm{Pd}$ conceived of the study, participated in its design and helped draft the 
manuscript. TN conceived of the study, participated in its design and helped draft the manuscript. All authors read and approved the final manuscript.

\section{Acknowledgements}

The study is funded by the Northern Territory Department of Health and Families, Darwin

\section{Author details}

${ }^{1}$ Wellbeing and Preventable Chronic Diseases Division, Menzies School of Health Research and School of Environmental and Life Sciences, Charles Darwin University, Darwin, Northern Territory, Australia. ${ }^{2}$ School of Environmental and Life Sciences, Charles Darwin University, Darwin, Northern Territory, Australia. ${ }^{3}$ Department of Head and Neck Surgery, Royal Darwin Hospital, Darwin, Northern Territory, Australia. ${ }^{4}$ Institute of Health \& Biomedical Innovation and School of Psychology and Counselling, Queensland University of Technology, Brisbane, Australia.

Received: 20 August 2012 Accepted: 9 October 2012

Published: 29 October 2012

\section{References}

1. Alcohol and Injury in Emergency Departments: Summary of the Report from the WHO Collaborative Study on Alcohol and Injuries. World Health Organization; 2007. http://www.who.int/substance_abuse/publications/ alcohol injury summary.pdf.

2. Ker K, Chinnock P: Interventions in the alcohol server setting for preventing injuries. Cochrane Database Syst Rev 2008, 16(3):CD005244.

3. National Health and Medical Research Council: Australian guidelines to reduce health risks from drinking alcohol. Canberra. Commonwealth of Australia: Australian Government; 2009.

4. Borges G, Mondragón L, Medina-Mora ME, Orozco R, Zambrano J, Cherpite C: A case-control study of alcohol and substance use disorders as risk factors for non-fatal injury. Alcohol Alcohol 2005, 40(4):257-262.

5. Cherpitel CJ, Ye Y: Alcohol-Attributable fraction for injury in the U.S General population: Data from the 2005 national alcohol survey. J Stud Alcohol Drugs 2008, 69(4):535-538.

6. Borges G, Cherpitel C, Mittleman M: Risk of injury after alcohol consumption: A case-crossover study in the emergency department. Soc Sci Med 2004, 58(6):1191-1200.

7. Lucas CE: The impact of street drugs on trauma care. J Trauma 2005 59(3 Suppl):S57-60. discussion S67-75.

8. London JA, Battistella FD: Testing for substance use in trauma patients: are we doing enough? Arch Surg 2007, 142(7):633-638

9. Macdonald S, Anglin-Bodrug K, Mann RE, et al: Injury risk associated with cannabis and cocaine use. Drug Alcohol Depend 2003, 72:99-115.

10. Cherpitel CJ, Bond J, Ye Y, et al: Multi-level analysis of causal attribution of injury to alcohol and modifying effects: data from two international emergency room projects. Drug Alcohol Depend 2006, 82:258-268.

11. Adams K, Sandy L, Smith L, Triglone B: Drug use monitoring in Australia (DUMA):2007 annual report on drug use among police detainees. In Research and public policy series no 93 Canberra: Australian Institute of Criminology; 2007. http://www.aic.gov.au/publications/current series/rpp/8199/rpp93.aspx

12. Collins D, Lapsley H: The costs of tobacco, alcohol and illicit drug abuse to Australian society in 2004/05. Canberra: Australian Government Department of Health and Ageing; 2008. Monograph Series No. 64.

13. Helps Y, Harrison J: Reported injury mortality of Aboriginal and Torres Strait Islander peoples in Australia, Canberra. Australian Institute of Health and Welfare; 2004:1997-2000. http://www.nisu.flinders.edu.au/pubs/reports/ 2004/injcat66.pdf.

14. Chikritzhs T, Pascal R: National Alcohol Indicators Bulletin No 6; 2004. http://ndri.curtin.edu.au/local/docs/pdf/naip/naip006.pdf.

15. Pascal R, Chikritzhs T, Gray D: Estimating alcohol-attributable mortality among Indigenous Australians: towards Indigenous-specific alcohol aetiologic fractions. Drug Alcohol Rev 2009, 28(2):196-200

16. Skov SJ, Chikritzhs TN, Li SQ, Pircher S, Whetton S: How much is too much? Alcohol consumption and related harm in the Northern Territory. Med J Aust 2010, 193(5):269-272.
17. You J, Guthridge S: Mortality, morbidity and health care costs of injury in the Northern Territory, 1991-2001. Health gains planning, Department of Health and Community Services Northern Territory; 2005. http://digitallibrary.health. nt.gov.au/dspace/bitstream/10137/60/1/injury_project_report.pdf.

18. Northern Territory Quarterly Crime \& Justice Statistics Issue SQ: Northern Territory of Australia, Department of Justice; 2009. http://www.nt.gov.au/ justice/policycoord/documents/statistics/28/QR\%20lssue\%2028\%20-\% 20Ebook.pdf.

19. Morgan A, McAtamney A: Key issues in alcohol-related violence. Canberra: Australian Institute of Criminology; 2009

20. Jayaraj R, Thomas M, Thomson V, Griffin C, Mayo L, Whitty M, Abbs P, Nagel $\mathrm{T}$ : High risk alcohol-related trauma among the Aboriginal and Torres Strait Islanders in the Northern Territory. Substance Abuse Treatment, Prevention, and Policy 2012, 7(1):33. 10.1186/1747-597X-7-33.

21. Thomas $M$, Jameson C: Facial trauma and post interventional quality of life in the Northern Territory, Australia. Int J Oral Maxillofac Surg 2007, 36(11):1081.

22. Barber JG, Punt J, Alberts J: Alcohol and power on Palm Island. Australian Journal of Social Issues 1988, 32:89-101.

23. Bertholet N, Daeppen J, Wietlisbach V, Fleming M, Burnand B: Reduction of Alcohol Consumption by Brief Alcohol Intervention in Primary Care: Systematic Review and Meta-analysis. Arch Intern Med 2005, 165(9):986-995.

24. Kaner E, Bland M, Cassidy P, Coulton S, Deluca P, Drummond C, Gilvarry E, Godfrey C, Heather N, Myles J, et al: Screening and brief interventions for hazardous and harmful alcohol use in primary care: a cluster randomised controlled trial protocol. BMC Public Health 2009, 9:287.

25. Soderstrom CA, DiClemente CC, Dischinger PC, Hebel JR, McDuff DR, Auman KM, Kufera JA: A controlled trial of brief intervention versus brief advice for at-risk drinking trauma center patients. J Trauma 2007, 62(5):1102-1111. discussion 1111-1102.

26. Whitlock EP, Polen MR, Green CA, Orleans T, Klein J: Behavioral counseling interventions in primary care to reduce risky/harmful alcohol use by adults:a summary of the evidence for the U.S. Preventive Services Task Force. Annintern Med 2004, 140:557-568.

27. Kaner EFS, Dickinson HO, Beyer FR, Campbell F, Schlesinger C, Heather N, Saunders JB, Burnand B, Pienaar ED: Effectiveness of brief alcohol interventions in primary care populations. Cochrane Database Syst Rev. 2007, CD004148. http://onlinelibrary.wiley.com/doi/10.1002/14651858. CD004148.pub3/pdf

28. Barrowclough C, Haddock G, Tarrier N, Lewis S, J M, O'Brien R, Schofield N, McGovern J: Randomised controlled trial of motivational interviewing cognitive behavior therapy, and family intervention for patients with comorbid schizophrenia and substance use disorders. Am J Psychiatry 2001, 158:1706-1713.

29. Bertholet N, Daeppen J, Wietlisbach V, Fleming M, Burnand B: Reduction of Alcohol Consumption by Brief Alcohol Intervention in Primary Care: Systematic Review and Meta-analysis. Arch Intern Med 2005, 165(9):986-995.

30. Bailie R, Si D, O'Donoghue L, Dowden M: Indigenous health: effective and sustainable health services through continuous quality improvement. MJA 2007, 186(10):525-527.

31. Bodenheimer T, Lorig K, Holman H, Grumbach K: Patient Self-management of Chronic Disease in Primary Care. JAMA 2002, 288(19):2469-2475.

32. Clarke S, Oades L, Crowe T, Deane F: Collaborative goal technology: theory and practice. Psychiatr Rehabil J 2006, 30(2):129-136.

33. Hickie I: An approach to managing depression in general practice. Medical Journal of Australia 2000, 173:106-110.

34. Coulton S, Watson J, Bland M, Drummond C, Kaner E, Godfrey C, Hassey A, Morton V, Parrott S, Phillips T, et al: The effectiveness and costeffectiveness of opportunistic screening and stepped care interventions for older hazardous alcohol users in primary care (AESOPS) - a randomised control trial protocol. BMC Health Serv Res 2008, 8:129.

35. Dawson DA, Grant BF, Stinson FS, Zhou Y: Effectiveness of the Derived Alcohol Use Disorders Identification Test (AUDIT-C) in Screening for Alcohol Use Disorders and Risk Drinking in the US General Population. Alcohol Clin Exp Res 2005, 29(5):844-854.

36. Diguiseppi C, Goss C, Xu S, Magid D, Graham A: Telephone screening for hazardous drinking among injured patients seen in acute care clinics: feasibility study. Alcohol Alcohol 2006, 41(4):438-445. 
37. Nagel T, Robinson G, Condon J, Trauer T: Approach to treatment of mental illness and substance dependence in remote Indigenous communities: results of a mixed methods study. Aust J Rural Health 2009 17(4):174-182.

38. Saunders JB, Aasland OG, Babor TF, De La Fuente JR, Grant M: Development of the Alcohol Use Disorders Identification Test (AUDIT): WHO Collaborative Project on Early Detection of Persons with Harmful Alcohol Consumption-II. Addiction 1993, 88(6):791-804.

39. Simon C, Katherine P, Martin B, Paul C, Mike C, Paolo D, Colin D, Eilish G, Christine $G$, Nick $H$, et al: Screening and brief interventions for hazardous alcohol use in accident and emergency departments: a randomised controlled trial protocol. BMC Health Serv Res 2009, 9(114). doi:10.1186/ 1472-6963-1189-1114.

40. Schlesinger CM, Ober C, McCarthy MM, Watson JD, Seinen A: The development and validation of the Indigenous Risk Impact Screen (IRIS): a 13-item screening instrument for alcohol and drug and mental health risk. Drug Alcohol Rev 2007, 26(2):109-117.

41. Bradley KA, Lapham GT, Hawkins EJ, Achtmeyer CE, Williams EC, Thomas RM, Kivlahan DR: Quality concerns with routine alcohol screening in VA clinical settings. J Gen Intern Med 2011, 26(3):299-306.

42. Proude $\mathrm{E}$, Britt $\mathrm{H}$, Valenti $\mathrm{L}$, Conigrave $\mathrm{K}$ : The relationship between selfreported alcohol intake and the morbidities managed by GPs in Australia. BMC Fam Pract 2006, 7(1):17.

43. Williams $R$, Vinson DC: Validation of a single screening question for problem drinking. J Fam Pract 2001, 50:307-312.

44. Bradley KA, Bush KR, Epler AJ, Dobie DJ, Davis TM, Sporleder JL, Maynard C, Burman ML, Kivlahan D: Two brief alcohol-screening tests from the Alcohol Use Disorders Identification Test (AUDIT): validation in a female veterans affairs patient population. Arch Intern Med 2003, 163:821-829.

45. Gordon AJ, Maisto SA, McNeil M, Kraemer KL, Conigliaro RL, Kelley ME, Conigliaro J: Three questions can detect hazardous drinkers. J Fam Pract 2001, 50:313-320.

46. Bush K, Kivlahan DR, McDonell MB, Fihn SD, Bradley KA: The AUDIT alcohol consumption questions (AUDIT-C): an effective brief screening test for problem drinking. Ambulatory Care Quality Improvement Project (ACQUIP). Arch Intern Med 1998, 158:1789-1795.

47. NHMRC Australian guidelines to reduce health risks from drinking alcohol: National Health \& Medical Research Council. Canberra; 2009. http://www. nhmrc.gov.au/files_nhmrc/publications/attachments/ds10-alcohol.pdf.

48. Dawe S, Loxton N, Hides L, Kavanagh D, Mattick R: Review of diagnostic screening instruments for alcohol and other drug use and psychiatric disorders. 2nd edition. Canberra: Publications Production Unit, Commonwealth Department of Health and Ageing; 2002.

49. Hides $L$, Dawe $S$, Young $R$, Kavanagh $D$ : The reliability and validity of the Severity of Dependence Scale for detecting cannabis dependence in psychosis. Addiction 2007, 102:35-40.

50. Hides L, Dawe S, Young RM, Kavanagh DJ: The reliability and validity of the Severity of Dependence Scale for detecting cannabis dependence in psychosis. Addiction 2007, 102(1):35-40.

51. Lawrinson P, Copeland J, Gerbera S, Gilmoura S: Determining a cut-off on the Severity of Dependence Scale (SDS) for alcohol dependence. Addictive Behaviours 2006, 32:1474-1479.

52. Sobell LC, Agrawal S, Annis H, Ayala-Velazquez H, Echeverria L, Leo Gl, Rybakowski JK, Sandahl C, Saunders B, Thomas S, et al: Cross-cultural evaluation of two drinking assessment instruments: alcohol timeline followback and inventory of drinking situations. Subst Use Misuse 2001 36(3):313-331.

53. Kessler RC, Andrews G, Colpe LJ, Hiripi E, Mroczek DK, Normand SL, Walters EE, Zaslavsky AM: Short screening scales to monitor population prevalences and trends in non-specific psychological distress. Psychol Med 2002, 32(6):959-976.

54. Hides L, Lubman DI, Devlin H, Cotton S, Aitken C, Gibbie T, Hellard M: Reliability and validity of the Kessler 10 and Patient Health Questionnaire among injecting drug users. Aust N Z J Psychiatry 2007, 41:166-168

55. Sunderland M, Slade T, Stewart G, Andrews G: Estimating the prevalence of DSM-IV mental illness in the Australian general population using the Kessler Psychological Distress Scale. Aust N Z J Psychiatry 2011, 45(10):880-889.
56. Kessler RC, Andrews G, Mroczek D, Ustun TB, Wittchen HU: The World Health Organisation Composite International Diagnostic Interview ShortForm (CIDI-SF). Int J Methods Psychiatr Res 1998, 7:171-185.

57. Nagel T, Thompson C: The central role of Aboriginal families in motivational counselling: family support and family 'humbug'. Australian Indigenous Health Bulletin 2010, 10(1). http://healthbulletin.org.au/articles/ the-central-role-of-aboriginal-families-in-motivational-counselling-familysupport-and-family-'humbug'/.

58. McCambridge J, Day M: Randomized controlled trial of the effects of completing the Alcohol Use Disorders Identification Test questionnaire on self-reported hazardous drinking. Addiction 2008, 103(2):241-248.

doi:10.1186/1472-6963-12-371

Cite this article as: Jayaraj et al:: Study Protocol: Screening and Treatment of Alcohol-Related Trauma (START) - a randomised controlled trial. BMC Health Services Research 2012 12:371.

\section{Submit your next manuscript to BioMed Central and take full advantage of:}

- Convenient online submission

- Thorough peer review

- No space constraints or color figure charges

- Immediate publication on acceptance

- Inclusion in PubMed, CAS, Scopus and Google Scholar

- Research which is freely available for redistribution 